\title{
AVALIAÇÃO SENSORIAL DE SUCO DE UVA CV. ISABEL EM CORTES COM DIFERENTES CULTIVARES ${ }^{1}$
}

\author{
ROGÉRIO DE SÁ BORGES², SANDRA HELENA PRUDÊNCIO ${ }^{3}$, SERGIO RUFFO ROBERTO4 \\ ADRIANE MARINHO DE ASSIS
}

RESUMO - As videiras americanas constituem a base varietal do suco de uva brasileiro, com destaque para a cultivar Isabel (Vitis labrusca). O objetivo do presente trabalho foi avaliar a aceitação de consumidores por meio de avaliação sensorial de sucos de uva de 'Isabel' combinados em cortes com sucos de outras cultivares. Foram preparadas em panela extratora seis amostras de suco de uva integral: Isabel (100\%); Isabel (80\%)+ Concord (20\%); Isabel (80\%) + BRS Rúbea (20\%); Isabel (80\%) + BRS Cora (20\%); Isabel $(80 \%)+$ BRS Violeta (20\%) e Isabel (80\%) + BRS Carmem (20\%). Foram avaliados o teor de sólidos solúveis totais, acidez total e $\mathrm{pH}$ das amostras. Para a avaliação sensorial, contou-se com cinquenta julgadores que avaliaram os atributos Cor, Aroma, Sabor, Corpo e Aceitação Global, usando uma escala hedônica de nove pontos. Tanto o suco puro de 'Isabel' como os cortes tiveram aceitação sensorial satisfatória. Com relação à cor, os cortes contendo 'BRS Rúbea', 'BRS Cora', 'BRS Violeta' e 'BRS Carmem' apresentaram as maiores médias, 7,98; 8,$10 ; 7,96$ e 8,22, respectivamente. Para os demais atributos, como aroma, sabor, corpo e aceitação global, os cortes de suco de 'Isabel' com outras cultivares não diferiram do suco puro dessa cultivar, 6,84; 6,56; 6,80 e 6,74, respectivamente. Na avaliação do consumidor, todas as cultivares avaliadas, com exceção da 'Concord', podem ser usadas em cortes com a 'Isabel', na proporção de 4:1, para melhorar a coloração do suco de uva, sem alterar as demais características.

Termos para indexação: teste de aceitação; Vitis labrusca, panela extratora, suco de uva.

\section{SENSORIAL EVALUATION OF GRAPE JUICE 'ISABEL’ BLENDINGS}

\begin{abstract}
The American vines represent the basis of the Brazilian grape juice, especially the cultivar Isabel (Vitis labrusca). The aim of this study was to evaluate the consumer acceptance through sensory evaluation of grape juice from 'Isabel' blended with juices from other grape varieties. Six samples of grape juice were prepared by the Welch process: Isabel $(100 \%)$, Isabel $(80 \%)+$ Concord $(20 \%)$, Isabel $(80 \%)+$ BRS Rúbea (20\%), Isabel (80\%) + BRS Cora (20\%), Isabel (80\%) + BRS Violeta (20\%), Isabel (80\%) + BRS Carmem (20\%). The total soluble solids content, the acidity and the $\mathrm{pH}$ of the samples were evaluated. Fifty assessors were used for the sensory evaluation, who evaluated the attributes color, aroma, flavor, body and global acceptance, by using a nine point hedonic scale. Either the pure juice of 'Isabel' or the blends had satisfactory sensorial acceptance. Regarding color, the blends containing BRS Rúbea, BRS Cora, BRS Violeta and BRS Carmem presented the highest mean sensory evaluation, 7.98; 8.10; 7.96; e 8.22, respectively. For other attributes such as aroma, flavor, body and global acceptance, the blends did not differ from the pure juice of Isabel variety, 6,84; 6,56; 6,80 e 6,74, respectively. According to the assessment of consumer, all the cultivars tested, except Concord, can be used in blends with Isabel, at a ratio of 4:1, to improve the color of grape juice, without changing the other characteristics.
\end{abstract}

Index terms: acceptance testing, Vitis labrusca, Welch process, grape juice.

\footnotetext{
${ }^{1}$ Resumo Sinfruit 073 - Simpósio Internacional de Fruticultura - Avanços na Fruticultura (17 a 21 Outubro)

${ }^{2}$ Engenheiro Agrônomo, Doutorando em Agronomia, Programa de Pós-graduação do Centro de Ciências Agrárias (CCA), Universidade Estadual de Londrina (UEL), Rod. Celso Garcia Cid, PR 445, Km 380. CEP 86051-990, CP 6001, Londrina-PR.

E-mail: rborges@cnpso.embrapa.br.

${ }^{3}$ Farmacêutica e Bioquímica, Dra. Professora Associada, Departamento de Ciência e Tecnologia de Alimentos. Universidade Estadual de Londrina (UEL). Rod. Celso Garcia Cid, PR 445, Km 380. CEP 86051-990, CP 6001, Londrina-PR. E-mail: sandrah@uel.br.

${ }^{4}$ Engenheiro Agrônomo, Dr. Professor Associado, Departamento de Agronomia/Fitotecnia, Universidade Estadual de Londrina (UEL), Bolsista do CNPq. Rod. Celso Garcia Cid, PR 445, Km 380. CEP 86051-990, CP 6001, Londrina-PR. E-mail: sroberto@uel.br. ${ }_{5}^{5}$ Engenheira Agrônoma, Dra. Professora Adjunta, Departamento de Agronomia/Fitotecnia, Universidade Estadual de Londrina (UEL), Bolsista do CNPq. Rod. Celso Garcia Cid, PR 445, Km 380. CEP 86051-990, CP 6001, Londrina-PR. E-mail: agroadri@ig.com.br.
} 


\section{INTRODUÇÃO}

Dependendo do processo de obtenção, o suco de uva pode ser classificado como suco de uva concentrado, reprocessado ou reconstituído, desidratado, integral e adoçado. O suco integral é obtido da uva por meio de processos tecnológicos adequados, sem a adição de açúcares e na sua constituição natural (BRASIL, 2004).

Pontes et al. (2010) avaliaram os atributos sensoriais e a aceitação dos diferentes tipos de sucos comerciais e verificaram que o suco integral apresentou o melhor equilíbrio entre os atributos doçura, amargor e adstringência, e apresentou também maior intenção de compra.

Rizzon (2006) descreveu que, na produção do suco de uva integral, um volume considerável das uvas é destinado para a produção de suco elaborado com um equipamento simples, denominado panela extratora. Nesse processo, o suco de uva é engarrafado a quente, em uma temperatura suficiente para garantir a estabilidade biológica e a conservação sem aditivos químicos.

Em muitos países de tradição vitícola, o suco é elaborado com uvas Vitis vinifera tanto de cultivares brancas quanto de tintas (THIBON et al., 2009). No entanto, o suco de uva brasileiro é elaborado principalmente com uvas do grupo das americanas e híbridas, sendo as cultivares Isabel, Bordô e Concord, todas de Vitis labrusca, a base para o suco brasileiro (TERRA et al., 2001).

A cultivar Isabel, pela grande disponibilidade de matéria-prima, é a responsável pelo maior volume de suco produzido no Brasil; no entanto, tem coloração e teor de açúcar abaixo do desejável (CAMARGO et al., 2010). Segundo Fenocchio (1973), para obtenção de melhor coloração, o suco de 'Isabel' deve ser cortado com sucos de outras cultivares.

Alguns materiais lançados nos últimos anos destacam-se pelos altos teores de açúcar e coloração que imprimem ao suco como a 'BRS Rúbea', 'BRS Cora', 'BRS Violeta' e 'BRS Carmem' (CAMARGO et al., 2010). Essas variedades podem ser utilizadas na elaboração de sucos puros (varietal), ou combinados com variedades tradicionais, como 'Isabel' e 'Concord' (cortes), no intuito de se melhorar a qualidade.

Para se mensurar a aceitação e a preferência dos consumidores com relação a um ou mais produtos, a escala hedônica estruturada de nove pontos é, provavelmente, o método afetivo mais utilizado devido à confiabilidade e à validade de seus resultados, bem como sua simplicidade em ser utilizada pelos provadores (VILLANUEVA et al., 2005).

Uma vez que a base varietal da indústria na- cional de suco de uva se encontra na cultivar Isabel, e, considerando algumas possibilidades de cortes possíveis com novos materiais para melhoria da qualidade, objetivou-se com o presente trabalho avaliar, em escala laboratorial, a aceitação e a preferência de consumidores por meio de testes de aceitação de sucos de uva de 'Isabel' cortados com sucos de outras cultivares.

\section{MATERIAIS E MÉTODOS}

As amostras de suco foram elaboradas a partir de uvas colhidas em janeiro de 2011, na área experimental do Centro Tecnológico da Corol Cooperativa Agroindustrial, localizado no município de Rolândia-PR.

Foram utilizadas as cultivares Isabel, Concord, BRS Rúbea, BRS Cora, BRS Violeta e BRS Carmem. O ponto de colheita das uvas foi determinado a partir do acompanhamento dos teores em açúcar e ácidos orgânicos das uvas. A partir do momento em que se observou uma estabilização da evolução da maturação, as uvas foram colhidas.

Os sucos foram elaborados pelo processo caseiro com o equipamento conhecido por panela extratora a vapor. Foi utilizada uma panela com capacidade para sete quilos e, após a extração, o suco foi engarrafado ainda quente, sendo, posteriormente, armazenado em geladeira para conservação.

Foram preparados 12,5 litros de suco de 'Isabel' mais 2,5 litros de cada uma das demais cultivares. Uma amostra de 2,5 litros de 'Isabel' (100\%) foi utilizada como testemunha e foram preparadas outras cinco amostras contendo 2,0 litros de suco de 'Isabel' (80\%) e 0,5 litro de suco de cada uma das demais cultivares $(20 \%)$, obtendo-se assim um total de seis amostras de 2,5 litros, na proporção de 4:1 (Tabela 1).

O teor de sólidos solúveis totais (SST) das amostras de suco foi determinado por meio de refratômetro digital de bancada com compensação de temperatura. As determinações do $\mathrm{pH}$ e da acidez total (AT) do suco foram realizadas em titulador potenciométrico digital. A acidez foi obtida por titulação do suco com solução padronizada de $\mathrm{NaOH}$ (CALEGUER et al., 2006). Os valores obtidos são apresentados na Tabela 2. Em virtude das diferenças entre os teores de SST encontradas nas amostras preparadas, decidiu-se pela padronização em $15^{\circ}$ Brix para que o teor de açúcar não interferisse na percepção dos julgadores. A padronização foi feita com a adição de sacarose. $\mathrm{O}$ valor final foi definido em função do maior valor encontrado antes da adição de açúcar e também considerando os teores de SST de 
sucos encontrados no mercado, assim como o valor mínimo estabelecido pela legislação brasileira, que é de $14^{\circ}$ Brix (BRASIL, 2000).

Para a avaliação do perfil de cada julgador do teste sensorial, foi aplicado um questionário (Quadro 1) a um grupo de 50 indivíduos (não treinados), constituído de alunos, professores e funcionários da Universidade Estadual de Londrina-PR. Neste questionário, foram avaliadas a faixa etária e a escolaridade dos entrevistados, a preferência e a frequência do consumo de suco de frutas.

$O$ teste de aceitação de atributos foi realizado em cabines individuais com iluminação luz do dia especial, no Laboratório de Análise Sensorial do Centro de Ciência Agrárias da Universidade Estadual de Londrina-PR. Foram avaliados os atributos cor, aroma, sabor, corpo e aceitação global das amostras de suco de uva, usando-se uma escala hedônica de nove pontos, com extremidades denominadas desgostei muitíssimo (1) e gostei muitíssimo (9) (Quadro 2) (VILLANUEVA et al., 2005).

As amostras foram servidas a $4^{\circ} \mathrm{C}$, em taças de acrílico transparente, na quantidade de $40 \mathrm{ml}$ de cada amostra. Cada julgador recebeu, em ordem aleatorizada, as seis amostras codificadas com números aleatórios de três dígitos. Água potável em temperatura ambiente foi servida aos julgadores para a limpeza da boca antes e entre as avaliações das amostras de suco de uva.

O delineamento experimental utilizado foi o de blocos completos casualizados, e os dados referentes à aceitação das seis amostras, avaliados pelos 50 julgadores, foram submetidos à análise de variância e teste de Tukey para comparação de médias, a 5\% de probabilidade.

\section{RESULTADOS E DISCUSSÃO}

Os resultados da avaliação do perfil dos julgadores referentes à faixa etária, ocupação, escolaridade, preferência e frequência de consumo foram separados entre homens e mulheres e são apresentados na Figura 1. A faixa etária predominante entre os provadores variou entre 15 e 30 anos. Com exceção da faixa entre 25 e 30 anos, com a maior parte de julgadores do sexo feminino, as faixas etárias superiores estiveram equilibradas entre homens e mulheres. Quanto à ocupação e escolaridade, predominaram alunos principalmente de terceiro grau. As maiores preferências quanto aos tipos de sucos de frutas perguntados tenderam para os sucos de Laranja e Uva, indicando que os julgadores eram consumidores potencias do suco em estudo. No entanto, também se verificou elevada preferência para os sucos de Maracujá e Abacaxi. Para essa pergunta, os provadores foram orientados a poderem escolher mais de uma alternativa. Para frequência de consumo, as respostas foram separadas em dois grupos, de acordo com o sexo, e foram elaborados histogramas com as frequências agrupadas em quatro classes. As maiores diferenças entre os dois grupos foram observadas nas classes de pouca e moderada frequência de consumo, sendo verificada para o grupo das mulheres maior frequência de consumo.

Os resultados referentes ao teste de aceitação dos sucos são apresentados na Tabela 3. Foram encontradas diferenças significativas entre julgadores, ou efeito de blocos. Isso é comum entre testes de consumidores, pois estes normalmente diferem entre si com relação ao grau de gostar ou desgostar das amostras avaliadas (VALIM et al., 2001).

As notas hedônicas médias para todos os atributos avaliados situaram-se entre 6,6 e 8,2, indicando que os julgadores gostaram dos sucos. Com relação à aceitação global a Aprovação (\% de notas entre $6 \mathrm{e}$ 9), Indiferença (\% de nota 5) e Rejeição (\% de notas entre 1 e 4) foram, respectivamente: 84,6 e $10 \%$ para Isabel; $82 ; 14$ e 4\% para Isabel + Concord; $92 ; 6$ e 2\% para BRS Cora; $92 ; 2$ e $6 \%$ para BRS Carmem e 84 ; 10 e $6 \%$ para BRS Violeta, e destacando o suco Isabel + BRS Rúbea, que apresentou $100 \%$ de aprovação.

Com relação à cor, verificaram-se diferenças significativas entre as diferentes amostras. Para esse atributo, as maiores médias foram obtidas com as amostras de suco da cultivar Isabel, em cortes com BRS Rúbea, BRS Cora, BRS Carmem e BRS Violeta. A amostra elaborada somente com a 'Isabel' e a combinada com 'Concord' obtiveram médias menores de aceitação pelo grupo de provadores. Segundo Camargo et al. (2010), a cultivar Isabel apresenta coloração e teor de açúcar abaixo do valor desejado. Para Matsuura et al. (2002), a cor é de fundamental importância, pois está ligada à atratividade para o consumidor.

Para os demais atributos, como aroma, sabor, corpo e aceitação global, não foram observadas diferenças significativas.

Camargo e Dias (1999) realizaram testes de avaliação sensorial de suco de uva puro de diferentes cultivares e verificaram médias superiores quanto à cor para a cultivar BRS Rúbea em comparação com a 'Isabel'.

Em uma equipe de análise sensorial realizada por Zanus et al. (2007 apud CAMARGO et al., 2010), amostras de suco puro de várias cultivares de videira foram avaliadas, e materiais como 'BRS Cora' e 'BRS Carmem' apresentaram performance superior à das cultivares Isabel e Concord quanto à aceitação global. 
$\mathrm{Na}$ proporção em que os cortes de 'Isabel' foram feitos com as demais variedades (4:1), não foi suficiente, na opinião dos julgadores, para alterar significativamente o nível de apreciação dos atributos, como aroma, sabor, corpo e aceitação global. Com exceção do corte de 'Isabel' com 'Concord', apenas as mudanças na cor, proporcionadas pelos cortes, obtiveram melhor aceitação dos julgadores. As amostras que se destacaram foram obtidas de cortes com materiais mais novos desenvolvidos com foco na melhoria dos principais atributos de qualidade, entre os quais a cor. O resultado do teste demonstra que, na proporção de corte avaliada, essa maior pigmentação do suco, obtida pelo melhoramento genético, foi mais bem aceita pelos julgadores quando comparada com a coloração dos sucos tradicionais, como o suco puro de 'Isabel' ou sua combinação com 'Concord'. Segundo Camargo e Maia (2004), o suco de uva elaborado com a cultivar Concord, ainda que se destaque como referência de aroma e sabor, também carece de matéria corante.

Cortes com uma proporção maior de outras cultivares poderiam proporcionar resultados distintos na análise sensorial e talvez diferenças significativas pudessem ser observadas também para outros atributos, proporcionando resultados melhores ou piores quanto à aceitação. No entanto, considerando a predominância absoluta da cultivar 'Isabel' nas principais regiões produtoras de suco de uva em relação a outros materiais, é pouco provável que, num primeiro momento, as empresas que produzem suco de uva utilizem como matéria-prima grandes volumes dessas cultivares.

Outro fator que deve ser levado em consideração é o fato de que o paladar do consumidor brasileiro está habituado ao suco da cultivar Isabel. Mudanças na matriz varietal devem ser vistas com cautela e exaustivamente avaliadas antes de se alterar o processo produtivo. Por mais que o melhoramento genético tenha contribuído para o desenvolvimento de cultivares capazes de produzir sucos de melhor qualidade e com melhor rendimento, a aceitação pelo consumidor deve sempre ser considerada sob risco de prejuízos financeiros para o setor.

TABELA 1 - Quantidades e proporções das amostras utilizadas dos cortes de suco de uva de 'Isabel com as diferentes cultivares. Londrina-PR, 2011.

\begin{tabular}{lccc}
\hline \multirow{2}{*}{ Cultivares } & \multicolumn{3}{c}{ Quantidades (L) } \\
\cline { 2 - 4 } & Isabel & Corte & Total \\
\hline 1 - Isabel (100\%) & 2,5 & 0,0 & 2,5 \\
2 - Isabel (80\%) + Concord $(20 \%)$ & 2,0 & 0,5 & 2,5 \\
3 - Isabel (80\%) + BRS Rúbea (20\%) & 2,0 & 0,5 & 2,5 \\
4 - Isabel (80\%) + BRS Cora (20\%) & 2,0 & 0,5 & 2,5 \\
5 - Isabel (80\%)+ BRS Violeta (20\%) & 2,0 & 0,5 & 2,5 \\
6 - Isabel (80\%) + BRS Carmem (20\%) & 2,0 & 0,5 & 2,5 \\
\hline Total & $\mathbf{1 2 , 5}$ & $\mathbf{2 , 5}$ & $\mathbf{1 5 , 0}$ \\
\hline
\end{tabular}

TABELA 2 - Resultados das análises químicas das amostras de suco de uva. Londrina-PR, 2011.

\begin{tabular}{lccc}
\hline Cultivares & pH & $\begin{array}{c}\text { SST } \\
\left({ }^{\circ} \text { Brix }\right)\end{array}$ & $\begin{array}{c}\text { AT } \\
(\%)\end{array}$ \\
\hline Isabel (100\%) & 3,20 & 13,5 & 0,77 \\
Isabel (80\%) + Concord (20\%) & 3,25 & 13,1 & 0,77 \\
Isabel (80\%)+ BRS Rúbea (20\%) & 3,25 & 14,0 & 0,76 \\
Isabel (80\%)+ BRS Cora (20\%) & 3,29 & 13,7 & 0,78 \\
Isabel (80\%)+ BRS Violeta (20\%) & 3,30 & 15,0 & 0,74 \\
Isabel (80\%)+ BRS Carmem (20\%) & 3,33 & 14,3 & 0,74 \\
\hline
\end{tabular}


TABELA 3 - Valores hedônicos médios para os atributos de cor, aroma, sabor, corpo e aceitação global obtidos no teste de aceitação das amostras de suco de uva. Londrina-PR, 2011.

\begin{tabular}{lccccc}
\hline \multirow{2}{*}{ Amostras de Suco } & \multicolumn{5}{c}{ Atributos } \\
\cline { 2 - 6 } & Cor & Aroma & Sabor & Corpo & Global \\
\hline Isabel 100\% & $7,42 \mathrm{~b}$ & $6,84 \mathrm{a}$ & $6,56 \mathrm{a}$ & $6,80 \mathrm{a}$ & $6,74 \mathrm{a}$ \\
Isabel + Concord & $7,38 \mathrm{~b}$ & $6,66 \mathrm{a}$ & $6,72 \mathrm{a}$ & $7,08 \mathrm{a}$ & $7,02 \mathrm{a}$ \\
Isabel + BRS Rúbea & $7,98 \mathrm{a}$ & $6,60 \mathrm{a}$ & $6,92 \mathrm{a}$ & $7,00 \mathrm{a}$ & $7,14 \mathrm{a}$ \\
Isabel + BRS Cora & $8,10 \mathrm{a}$ & $6,90 \mathrm{a}$ & $7,00 \mathrm{a}$ & $7,24 \mathrm{a}$ & $7,28 \mathrm{a}$ \\
Isabel + BRS Carmem & $7,96 \mathrm{a}$ & $6,84 \mathrm{a}$ & $7,02 \mathrm{a}$ & $7,08 \mathrm{a}$ & $7,30 \mathrm{a}$ \\
Isabel + BRS Violeta & $8,22 \mathrm{a}$ & $6,72 \mathrm{a}$ & $6,74 \mathrm{a}$ & $6,94 \mathrm{a}$ & $6,96 \mathrm{a}$ \\
\hline CV (\%) & 10,67 & 16,84 & 19,01 & 13,74 & 15,70 \\
\hline
\end{tabular}

Médias seguidas de mesma letra na vertical não diferem significativamente, ao nível de $5 \%$ ( $<<0,05)$, segundo o teste de Tukey.

\section{DADOS PESSOAIS:}

NOME:

TELEFONE: e-mail:

\section{FAIXA ETÁRIA:}

( ) 15-25 anos

( ) 25-30 anos

( ) 30-35 anos

( ) 35-50 anos

( ) acima de 50 anos

\section{OCUPAÇÃO:}
( ) aluno
( ) funcionário
( ) professor
( ) outro

SEXO:
( ) masculino
( ) feminino

GOSTA DE SUCOS? ( ) sim ( ) não
SABOR:
( ) laranja
( ) uva
( ) maracujá
( ) abacaxi

\section{ESCOLARIDADE}
( ) $1^{\circ}$ grau
( ) $2^{\circ}$ grau
( ) $3^{\circ}$ grau
( ) outro

\section{FREQUENCIA COM QUE CONSOME SUCO DE UVA:}

( ) nunca

( ) ocasionalmente:

( ) moderadamente:

vezes por ano

( ) frequentemente: vezes por mês vezes por semana

QUADRO 1 - Questionário utilizado na avaliação do perfil de provadores no teste de aceitação. 
Nome:

Data:

Avalie cada uma das amostras codificadas, da esquerda para a direita. Use a escala abaixo para indicar o quanto você gostou ou desgostou de cada atributo (cor, aroma, sabor, corpo e global) em cada amostra.

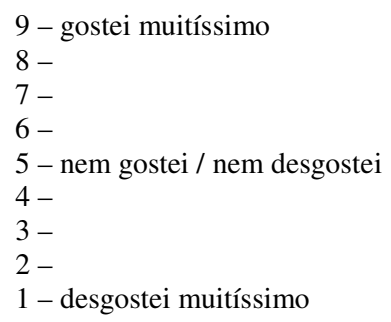

\begin{tabular}{|l|l|l|l|l|l|l|}
\hline Amostras & no: & no: & no: & no: & no: & no: \\
\hline Cor & & & & & & \\
\hline Aroma & & & & & & \\
\hline Sabor & & & & & & \\
\hline Corpo & & & & & & \\
\hline Global & & & & & & \\
\hline
\end{tabular}

Comentários:

QUADRO 2 - Ficha de avaliação usada para o teste de aceitação das amostras de suco de uva.
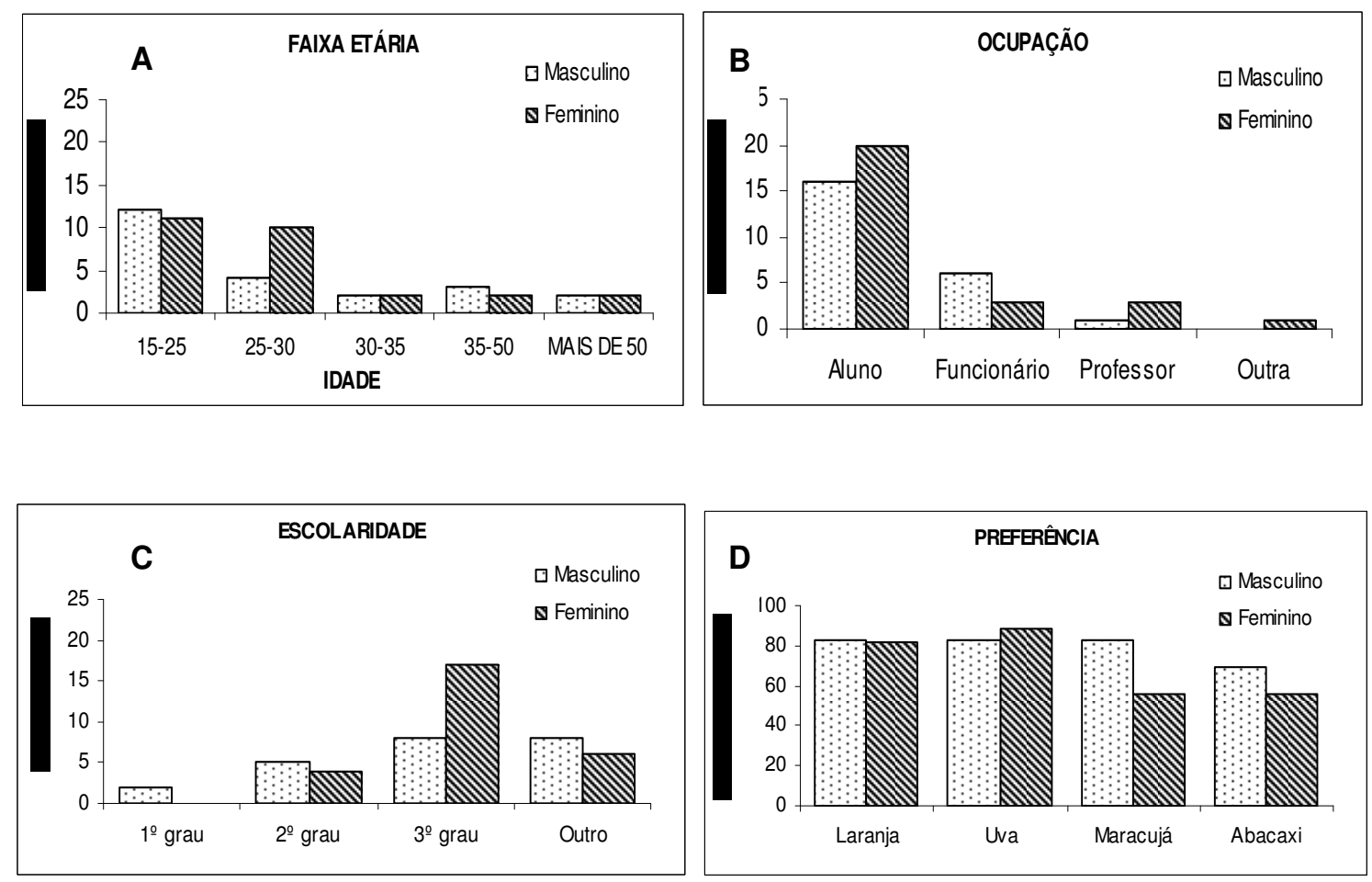

continua... 
continuação
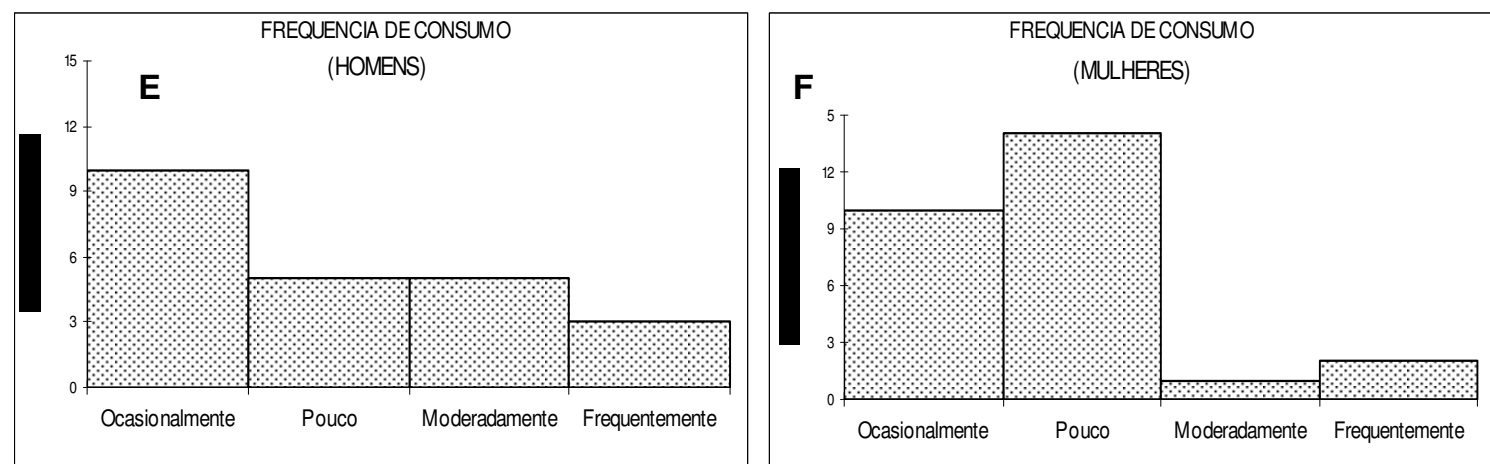

FIGURA 1 -Resultados da avaliação do perfil dos provadores referentes à faixa etária (A), ocupação (B), escolaridade (C), preferência (D) e frequência de consumo (E e F). Londrina-PR, 2011.

\section{CONCLUSÕES}

1. O suco puro de 'Isabel' e os cortes com as cultivares Concord, BRS Rúbea, BRS Cora, BRS Violeta e BRS Carmem tiveram aceitação sensorial satisfatória.

2. Na avaliação do consumidor, todas as cultivares avaliadas, com exceção da 'Concord', podem ser usadas em cortes com a 'Isabel', na proporção de 4:1, para melhorar a coloração do suco de uva sem alterar as demais características.

3. Os cortes de suco de 'Isabel' com outras cultivares, na proporção de 4:1, não diferiram do suco puro dessa cultivar quanto à aceitação dos atributos de aroma, sabor, corpo e global.

\section{AGRADECIMENTOS}

Os autores agradecem à Universidade Estadual de Londrina, à Corol Cooperativa Agroindustrial e ao Conselho nacional de Desenvolvimento Científico e Tecnológico.

\section{REFERÊNCIAS}

BRASIL. Ministério da Agricultura, Pecuária e Abastecimento. Instrução normativa ${ }^{\circ} 01$, de 7 de janeiro de 2000. Regulamento técnico geral para fixação dos padrões de identidade e qualidade para suco de fruta. Disponível em: $<$ http://www.agricultura. gov.br/legislacao>. Acesso em: 25 maio 2011.

BRASIL. Portaria $\mathrm{n}^{\circ}$ 55, de 27 de julho de 2004. Normas referentes à complementação dos padrões de identidade e qualidade do vinho e dos derivados da uva e do vinho. Disponível em: $<$ http://www.agricultura.gov.br/legislacao>. Acesso em: 26 maio 2011.
CALEGER, V. F.; TOFFOLI, C.; BENASSI, M. T. Avaliação da aceitação de preparados sólidos comerciais para refresco sabor laranja e correlação com parâmetros físico-químicos. Semina: Ciências Agrárias, Londrina, v. 27, n 4, p.587-598, 2006.

CAMARGO, U. A.; DIAS, M. F. BRS Rúbea. Bento Gonçalves: Embrapa Uva e Vinho, 1999. 4 p. (Comunicado Técnico, 33).

CAMARGO, U. A.; MAIA, J. D. G. BRS Cora: nova cultivar de uva para suco, adaptada a climas tropicais. Bento Gonçalves: Embrapa Uva e Vinho, 2004. 4p. (Comunicado Técnico, 53).

CAMARGO, U. A.; MAIA, J. D. G.; RITSCHEL, P. S. Novas cultivares brasileiras de uva. Bento Gonçalves. Embrapa Uva e Vinho, 2010. 64 p.

FENOCCHIO, P. Competição da matéria-prima para elaboração de suco de uva. Pesquisa Agropecuária Brasileira, Brasília, v.8, n.8, p. 263-266, 1973.

MATSUURA, F.C.A.U.; CARDOSO, R.L.; RIBEIRO, D.E. Qualidade sensorial de frutos de híbridos de bananeira cultivar Pacovan. Revista Brasileira de Fruticultura, Jaboticabal, v.24, n.1, p.263-266, 2002.

PONTES, P. R. B.; SANTIAGO, S. S.; SZABO, T. N.; TOLEDO, L. P.; GOLLUCKE, A. P. B. Atributos sensoriais e aceitação de sucos de uva comerciais. Ciência e Tecnologia de Alimentos, Campinas, v. 30, n. 2, p. 313-318, 2010.

RIZZON, L.A.; LINK, M. Composição do suco de uva caseiro de diferentes cultivares. Ciência Rural, Santa Maria, v.26, n. 2, p.689-692, 2006. 
TERRA, M. M.; POMMER, C. V.; PIRES, E. J. P.; RIBEIRO, I.J.A.; GALLO, P.B.; PASSOS, I.R.S. Produtividade de cultivares de uva para suco sobre diferentes porta-enxertos IAC em Mococa-SP. Revista Brasileira de Fruticultura, Jaboticabal, v.23, n.2, p.382-386. 2001.

THIBON, C.; DUBOURDIEUA, D.; DARRIETA, P.; TOMINAGAA, T. Impact of noble rot on the aroma precursor of 3-sulfanylhexanol content in Vitis vinifera L. cv Sauvignon blanc and Semillon grape juice. Food Chemistry, London, v.114, n.4, p.1359-1364, 2009.
VALIM, M.F.; MARCELLINI, P.S.; CAVALHEIRO, S.; DEMARCHI, R.;SERAFIM, E. Preference Mapping to asses consumers orange juice preference. Fruit Processing, Schonborn, v.3, n.3, p. 90-94, 2001.

VILLANUEVA, N.D.M.; PETENATE, A.J.; DA SILVA, M.A.A.P. Perfomance of hibrid hedonic scale as compared to the traditional hedonic, self-adjusting and ranking scales. Food Quality and Preference, Oxford, v.16, n.8, p.691-703, 2005. 